\title{
DUAL-CONTROL MISSILE GUIDANCE: A SIMULATION STUDY
}

\author{
Witold BuŻantowicz, Jan Pietrasieński \\ Military University of Technology, Faculty of Mechatronics and Aerospace, Warsaw, Poland \\ e-mail: witold.buzantowicz@wat.edu.pl; jan.pietrasienski@wat.edu.pl
}

\begin{abstract}
Theoretical considerations and a simulation study concerning description and analysis of new autopilot structures and issues relating to conversion of guidance commands into deflections of a complex system of control surfaces aimed at minimizing the miss distance value are presented in this paper. Due to nonlinear and nonstationary nature of the phenomena associated with the guidance process, a significant role is assigned to simulation studies. A comparative analysis has been made of the guidance processes of three models of a dual-control missile, which differ in terms of formulae implemented in the control command converters.
\end{abstract}

Keywords: dual-control missile, autopilot, guidance, miss distance

\section{Introduction}

Recent years have seen a significant increase in the possibilities offered by the air attack, both in terms of the impact on the enemy and the tactics used. Common use is made of jamming, anti-missile maneuvers, long-distance guided glide bombs and anti-radiation missiles. The intensive development of aircraft as well as maneuvering and aeroballistic missiles, and the increasingly common use of unmanned aircraft systems on today's battlefields, demand that ways are found to counteract these threats. Areas in which progress has been made include solutions for producing the lift force and methods of controlling anti-aircraft missiles. In particular, this concerns the design of dual-control missiles which have sets of aerodynamic fins both in front of and behind the missile center of mass. The aim of these solutions is to extend maneuvering possibilities of the missiles, thereby to increase the effectiveness of control during the terminal guidance phase.

Dual-control missiles were subjected to scientific analysis in the early 1990s (Hull et al., 1990; Ochi et al., 1991). Research in this area has been carried out by centers in, for example, Israel (Idan et al., 2007; Shima and Golan, 2006, 2007), the United States (Schroeder, 2001; Shtessel and Tournes, 2009), China (Hua et al., 2016; Yan and Ji, 2012), Japan (Ochi, 2003) and Spain (Ibarrondo and Sanz-Aránguez, 2016). These studies have largely concentrated on the optimum distribution of signals between control channels with attention being given both to problems of minimizing the miss distance and to general strategies for controlling and guiding missiles to aerial targets.

The high maneuverability of modern airstrike weapons enables them to react effectively to fire, including through anti-missile maneuvers. This situation is particularly disadvantageous during the terminal guidance phase, as it requires a very rapid reaction on the part of the missile, involving significant rotations of its airframe. This disturbs working conditions of the seeker, increasing dynamic errors of the coordinate determination system (Grycewicz et al., 1984; Siouris, 2004; Yanushevsky, 2007; Gapiński and Krzysztofik, 2014). These errors are incorporated into the missile control commands, leading to increased miss distances and, thus, to reduced effectiveness of fire against the target. The minimization of miss distances is especially critical against ballistic missiles, which are generally destroyed by a direct hit, using the kinetic energy of 
the guided missile. In these situations, the miss distance cannot exceed geometrical dimensions of the target.

It, therefore, becomes necessary to seek for solutions enabling stabilization of the operating conditions of the on-board seeker. Every change in the angle of attack and the use of aerodynamic control moment to produce rotation of the airframe achieved through deflection of the fins brings about transitional processes in the system that have an adverse effect on the operating conditions of the seeker installed in the nose section.

The use of a dual-control aerodynamic scheme is interesting in view of the increased technical control possibilities offered and, thus, the extended possibilities available for the guidance process itself. For example, the sets of fins in the control planes may deflect in the same direction ("divert mode") or in opposite directions ("opposite mode") - that is, the angles of deflection of the control surfaces located in front of and behind the center of mass of the missile airframe may have either the same or different signs.

This study focuses on issues related to improving the operating conditions of the seeker installed on an anti-aircraft missile. Specifically, the terminal phase of the guidance of the missile to an aerial target - the phase of particular relevance to effective combating of modern highly maneuverable air attacks.

The structure of the paper is as follows. In Section 2, a mathematical model is given for the dynamics of dual-control missile flight. The models of the elements making up the guidance loop are presented in Section 3. In Sections 4 and 5, formulae are proposed for the conversion of control commands, with selected simulation results. Conclusions and closing remarks constitute the final Section.

\section{Dual-control missile dynamics model}

We consider a cruciform, roll-stabilized missile with dual controls located in front of and behind the center of mass (Fig. 1).

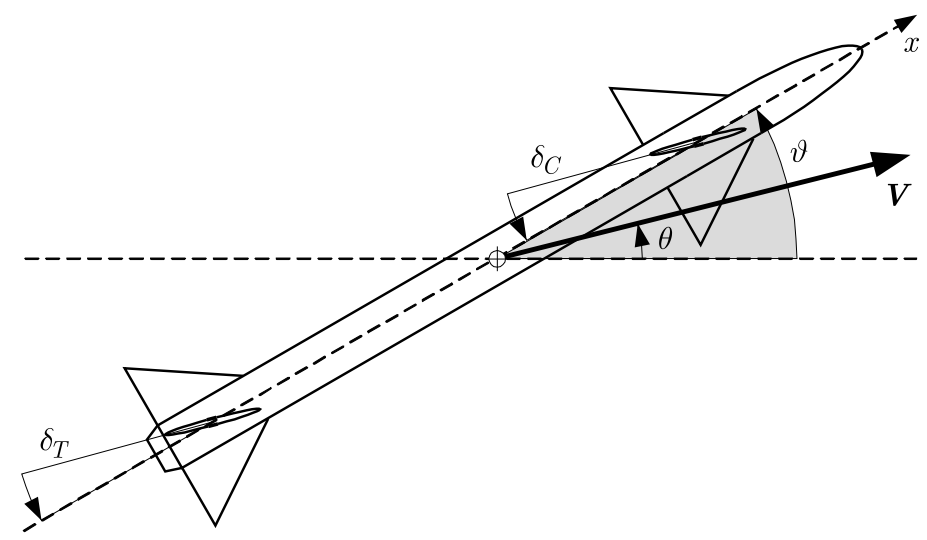

Fig. 1. Dual-control missile configuration

The motion of the missile is considered independently in the control planes. Selected questions will be addressed in the case of motion in the pitch plane.

The dynamics of the missile in the pitch plane is described by the following system of equations

$$
\dot{\mathbf{x}}(t)=\mathbf{A} \mathbf{x}(t)+\mathbf{B q}(t) \quad \mathbf{y}(t)=\mathbf{C x}(t)
$$

where $\mathbf{x}$ is a state vector

$$
\mathbf{x} \in \mathbb{R}^{5 \times 1}: \quad \mathbf{x}=\left[\begin{array}{lllll}
\theta & \omega & \vartheta & \delta_{C} & \delta_{T}
\end{array}\right]^{\mathrm{T}}
$$


$\mathbf{y}$ is a vector of output signals

$$
\mathbf{y} \in \mathbb{R}^{2 \times 1}: \quad \mathbf{y}=\left[\begin{array}{ll}
\omega & \vartheta
\end{array}\right]^{\mathrm{T}}
$$

and $\mathbf{q}$ is a control vector

$$
\mathbf{q} \in \mathbb{R}^{5 \times 1}: \quad \mathbf{q}=\left[\begin{array}{lllll}
0 & 0 & 0 & \delta_{C}^{c o m} & \delta_{T}^{c o m}
\end{array}\right]^{\mathrm{T}}
$$

The symbols in equations (2.2)-(2.4) have the following meanings: $\theta$ is the pitch angle of the missile velocity vector; $\omega$ is the angular rate of the missile airframe; $\vartheta$ is the pitch angle of the missile airframe; $\delta_{C}^{c o m}$ and $\delta_{C}$ are the commanded and actual deflections of the canards; $\delta_{T}^{\text {com }}$ and $\delta_{T}$ are the commanded and actual deflections of the tail fins.

The matrices $\mathbf{A}, \mathbf{B}$ and $\mathbf{C}$ are defined as follows

$$
\begin{aligned}
\mathbf{A} \in \mathbb{R}^{5 \times 5}: & \mathbf{A}=\left[\begin{array}{ccccc}
-A_{1} & 0 & A_{1} & A_{5}^{C} & A_{5}^{T} \\
A_{3} & -A_{4} & -A_{3} & -A_{2}^{C} & -A_{2}^{T} \\
0 & 1 & 0 & 0 & 0 \\
0 & 0 & 0 & -1 / \tau_{C} & 0 \\
0 & 0 & 0 & 0 & -1 / \tau_{T}
\end{array}\right] \\
\mathbf{B} \in \mathbb{R}^{5 \times 5}: & \mathbf{B}=\left[\begin{array}{lllll}
0 & 0 & 0 & 0 & 0 \\
0 & 0 & 0 & 0 & 0 \\
0 & 0 & 0 & 0 & 0 \\
0 & 0 & 0 & 1 / \tau_{C} & 0 \\
0 & 0 & 0 & 0 & 1 / \tau_{T}
\end{array}\right] \\
\mathbf{C} \in \mathbb{N}^{2 \times 5}: & \mathbf{C}=\left[\begin{array}{lllll}
0 & 1 & 0 & 0 & 0 \\
0 & 0 & 1 & 0 & 0
\end{array}\right]
\end{aligned}
$$

where $\tau_{C}$ and $\tau_{T}$ are the time constants for the canards and tail fin servos. The coefficients of the matrix $\mathbf{A}$ take the following forms

$$
\begin{aligned}
A_{1} & =\frac{\rho V}{2 m} f_{1}(M, \alpha) & & \\
A_{2}^{C} & =\frac{\rho V^{2}}{2 I} f_{2}^{C}\left(M, \delta_{C}\right) & A_{2}^{T} & =\frac{\rho V^{2}}{2 I} f_{2}^{T}\left(M, \delta_{T}\right) \\
A_{3} & =\frac{\rho V^{2}}{2 I} f_{3}(M, \alpha) & A_{4} & =\frac{\rho V}{2 I} f_{4}(M, \omega) \\
A_{5}^{C} & =\frac{\rho V}{2 m} f_{5}\left(M, \delta_{C}\right) & A_{5}^{T} & =\frac{\rho V}{2 m} f_{5}\left(M, \delta_{T}\right)
\end{aligned}
$$

where $V=|\mathbf{V}|$ is the module of the missile velocity vector, $\rho$ is the air density, and $\alpha \equiv \vartheta-\theta$ is the airframe angle of attack. Nonlinear functions $f_{i}(\cdot), i \in\{1, \ldots, 5\}$ describe the missile aerodynamics. These depend on, among others, geometric parameters of the airframe, Mach's number $M$, and values of the variables $\alpha, \omega, \delta_{C}$, and $\delta_{T}$. They have a complex form whose derivation lies outside the scope of this work. The polynomial approximations $\widehat{f}_{i}(\cdot), i \in\{1, \ldots, 5\}$ are taken for $M>1.15$, and the following are assumed to hold

$$
\begin{array}{ll}
f_{1}(M, \alpha) \approx S_{B} \widehat{f}_{1} & \\
f_{2}^{C}\left(M, \delta_{C}\right) \approx S_{C} l_{C} \widehat{f}_{2}^{C} & f_{2}^{T}\left(M, \delta_{T}\right) \approx S_{T} l_{T} \widehat{f}_{2}^{T} \\
f_{3}(M, \alpha) \approx S_{B} l_{B} \widehat{f}_{3} & f_{4}(M, \omega) \approx S_{B} l_{B}^{2} \widehat{f}_{4} \\
f_{5}\left(M, \delta_{C}\right) \approx S_{C} \widehat{f}_{5} & f_{5}\left(M, \delta_{T}\right) \approx S_{T} \widehat{f}_{5}
\end{array}
$$


where $S_{B}, S_{C}, S_{T}$ and $l_{B}, l_{C}, l_{T}$ are the characteristic surfaces and linear characteristic dimensions of the airframe, canards, and tail fins, respectively. The system of equations (2.1) is supplemented with the kinematic equation

$$
a_{a v}=V \dot{\theta}
$$

where $a_{a v}$ is the available acceleration relative to the normal of the missile velocity vector, and $V$ is the module of that vector.

The gravitational force acting on the missile during its flight is treated as an external signal the compensation of which is made in the numerical calculations by means of the adjustment

$$
\dot{\theta}_{G}=-\frac{g}{V} \cos \theta
$$

where $g=9.81 \mathrm{~m} / \mathrm{s}^{2}$ is the gravitational acceleration.

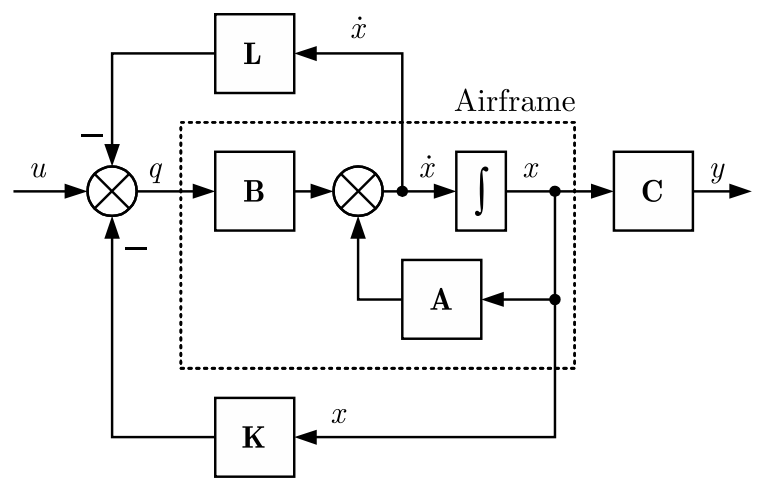

Fig. 2. Block diagram of the dual-control missile stabilization system in the pitch plane

A system for stabilization of the static and dynamic characteristics of the airframe is applied in the form of feedback loops, based on the angular rate and linear acceleration (Fig. 2). Consequently, a vector-matrix equation is obtained to describe motion of the dual-control missile in the pitch plane, taking account the stabilization system

$$
\dot{\mathbf{x}}=(\mathbf{I}+\mathbf{B L})^{-1}(\mathbf{A}-\mathbf{B K}) \mathbf{x}+(\mathbf{I}+\mathbf{B L})^{-1} \mathbf{B u}
$$

where $\mathbf{I}$ is the identity matrix, and $\mathbf{u}$ is the control command vector

$$
\mathbf{u} \in \mathbb{R}^{5 \times 1}: \quad \mathbf{u}=\left[\begin{array}{c}
\mathbf{0}^{3 \times 1} \\
\mathbf{u}_{\kappa}
\end{array}\right] \wedge \mathbf{u}_{\kappa}=\left[\begin{array}{c}
\kappa_{C} \\
\kappa_{T}
\end{array}\right]
$$

where, in turn, $\kappa_{C}$ is the command applied to the canards, $\kappa_{T}$ is the command applied to the tail fins, and $\mathbf{K}$ and $\mathbf{L}$ are matrices in the feedback paths

$$
\mathbf{K}, \mathbf{L} \in \mathbb{R}^{5 \times 5}: \quad \mathbf{K}=\left[\begin{array}{ccccc}
0 & 0 & 0 & 0 & 0 \\
0 & 0 & 0 & 0 & 0 \\
0 & 0 & 0 & 0 & 0 \\
0 & K_{C} & 0 & 0 & 0 \\
0 & K_{T} & 0 & 0 & 0
\end{array}\right] \quad \mathbf{L}=\left[\begin{array}{ccccc}
0 & 0 & 0 & 0 & 0 \\
0 & 0 & 0 & 0 & 0 \\
0 & 0 & 0 & 0 & 0 \\
L_{C} & 0 & 0 & 0 & 0 \\
L_{T} & 0 & 0 & 0 & 0
\end{array}\right]
$$

where $K_{C}, K_{T}, L_{C}$ and $L_{T}$ are the gains of component control signals for the canards and the tail fins. 


\section{Homing loop model}

The system under consideration is a homing system based on a proportional navigation method in which control signals are dependent on parameters of the line of sight (Siouris, 2004; Yanushevsky, 2007; Zarchan, 2012)

$$
a_{r e q}=n V_{C} \dot{\lambda}
$$

where $a_{r e q}$ is the required acceleration, $n$ is the navigational constant, $V_{C}$ is the closing velocity and $\dot{\lambda}$ is the angular rate of the line of sight.

A simplified block diagram of the missile homing system is shown in Fig. 3. The loop elements consist of the target, a seeker with a system for determining the target coordinates (TCD), an autopilot system (AP) and actuators.

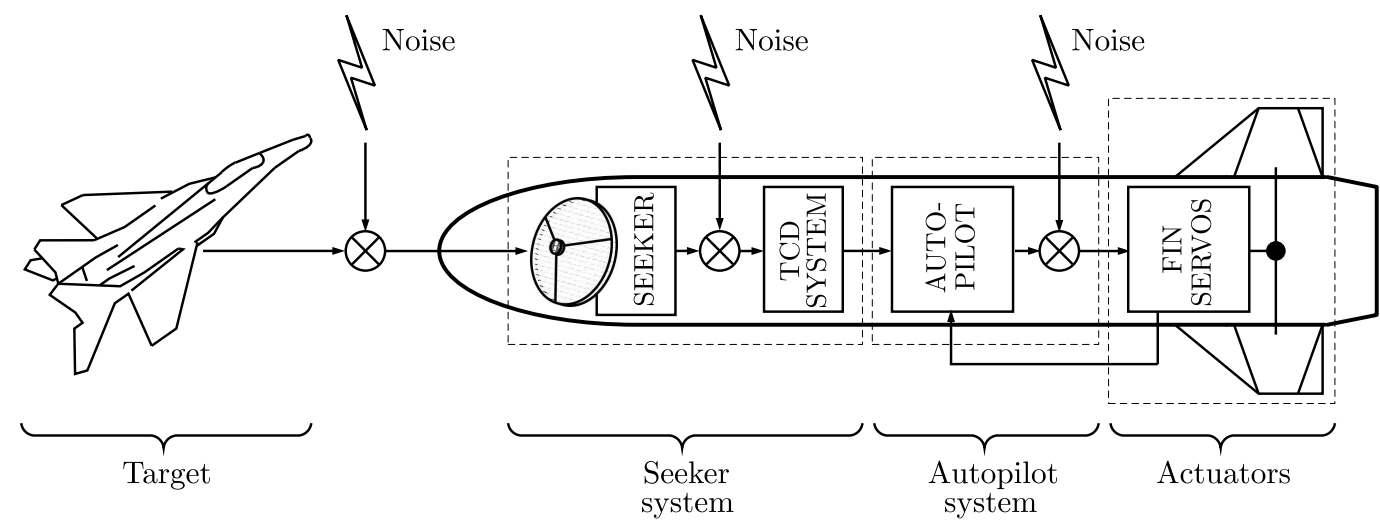

Fig. 3. Block diagram of the missile homing loop

The aerial target is modelled as a mass point whose motion is described by the vector $\mathbf{E}$

$$
\mathbf{E} \in \mathbb{R}^{1 \times 4}: \quad \mathbf{E}=\left[\begin{array}{llll}
x_{E} & y_{E} & \theta_{E} & V_{E}
\end{array}\right]
$$

where $x_{E}$ and $y_{E}$ are the coordinates of the instantaneous position of the target, $\theta_{E}$ is the pitch angle of the target velocity vector, and $V_{E}$ is the module of the target velocity vector. The changes in the target velocity and the pitch angle are defined by

$$
\dot{V}_{E}=a_{E}^{L} \quad \dot{\theta}_{E}=\frac{1}{V_{E}} a_{E}
$$

where $a_{E}^{L}$ is the longitudinal acceleration and $a_{E}$ is the lateral acceleration in the pitch plane. The changes in the accelerations $a_{E}^{L}$ and $a_{E}$ are approximated by the first-order dynamics

$$
\dot{a}_{E}^{L}=\frac{\kappa_{E}^{L}-a_{E}^{L}}{\tau_{E}} \quad \dot{a}_{E}=\frac{\kappa_{E}-a_{E}}{\tau_{E}}
$$

where $\kappa_{E}^{L}$ and $\kappa_{E}$ are the commanded accelerations and $\tau_{E}$ is the time constant. The commanded accelerations are subject to the following restrictions

$$
\left|\kappa_{E}^{L}\right| \leqslant \bar{\kappa}_{E}^{L} \quad\left|\kappa_{E}\right| \leqslant \bar{\kappa}_{E}
$$

The angle of the line of sight is given by

$$
\lambda=\arctan \frac{y_{E}-y}{x_{E}-x}
$$

where the coordinates $x$ and $y$ describe the instantaneous position of the missile. 
The seeker supplies data to the coordinate determination system. A significant problem relating to the homing process is the determination of the current angular rate of the line of sight as determination of the position of the target is hampered by the fact that the motion of the missile airframe affects the operation of the coordinate determination system. The signal returned by the seeker contains information about the angular rate of the line of sight distorted by the inertia of the system (Grycewicz et al., 1984) via a signal proportional to the angular acceleration of the missile airframe, and by generally understood measurement errors

$$
s \lambda_{m}(s)=\frac{k_{S}}{\tau_{S} s+1}\left[s \lambda(s)+s \Delta \lambda(s)+s^{2} \xi \vartheta(s)\right]
$$

where $\xi$ is the acceleration gain factor, $k_{S}$ and $\tau_{S}$ are the gain and the time constant of the seeker drives, and $\Delta \lambda$ denotes the sum of the error signals occurring in the measuring path.

The sector of observation of the seeker's antenna is subject to the following restriction

$$
\left|\vartheta-\lambda_{m}\right| \leqslant \zeta
$$

\section{Control command equations}

Under the classical approach, the following control equation may be applied for a missile guided using a method of proportional navigation (Grycewicz et al., 1984)

$$
\kappa=\eta_{1} \dot{\lambda}+\eta_{2} \ddot{\lambda}
$$

where $\kappa$ is the control command, $\eta_{1}$ is a navigation function dependent on time and mass-geometrical characteristics of the missile, and $\eta_{2}$ is a variable related to compensation of the errors resulting from the airframe dynamics. Expression (4.1) is insufficient to perform the guidance process of a dual-control missile, as it does not take into account the additional possibilities offered by the dual-control system - in particular, it does not contain a rule for selecting the control mode.

During the guidance of a missile to the target, it is desirable that

$$
\omega=\dot{\theta}=n \dot{\lambda}
$$

namely, that the angular rate of the airframe should be close to the angular rate of the missile velocity vector and should not depend on the dynamics of the missile itself. This requirement is the basis for the formulation of the quantity index

$$
J=E\left\{\int_{0}^{t_{f}}(\omega-\dot{\theta})^{2} d t\right\}
$$

where $E\{\cdot\}$ is the mean operator and $t_{f}$ is the final time. The value of the expression in (4.3) depends on the system ability to reduce the effect of components related to the dynamics of the missile airframe. This value will be smaller when the transitional processes occurring during guidance are shorter and less oscillatory. Figure 4a shows graphs of the angular rates of a dual-control missile airframe normalized to the final value, as they respond to step deflections of the fins (in the divert or opposite mode). The diagram shows the divert mode to be a more desirable control method in terms of the course of the process. A fundamental drawback, however, is that the accelerations available in the divert mode are many times smaller than those available in the opposite mode (Fig. 4b).

Based on Eq. (4.1) and the conditions described above, two forms of the conversion equations have been formulated for the mathematical model of the dual-control missile given by Eq. (2.10). 
(a)

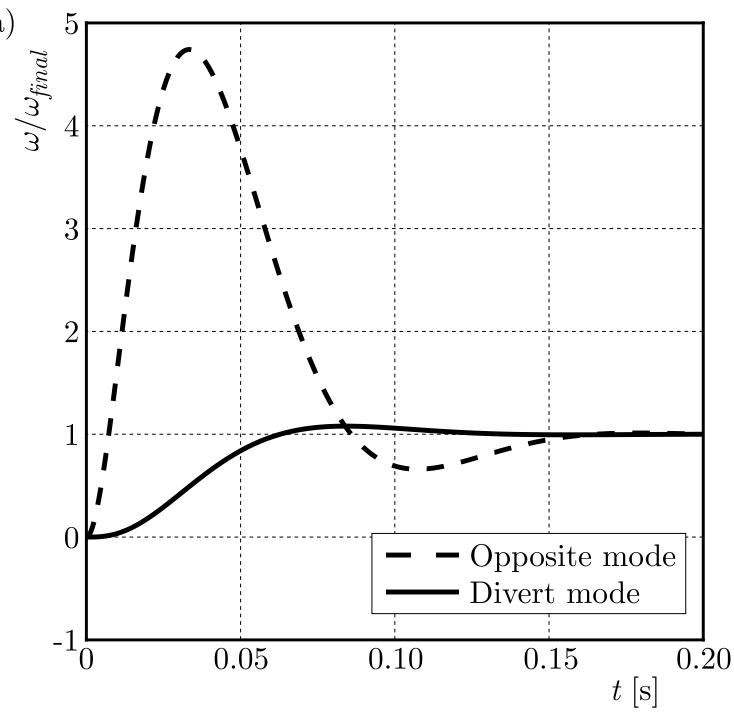

(b)

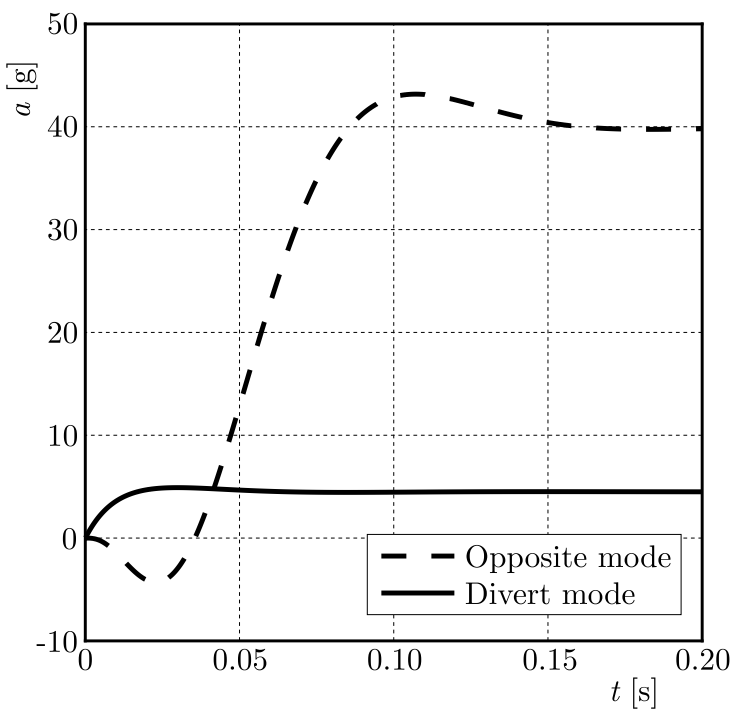

Fig. 4. Normalized angular rates (a) and normal accelerations (b) of the missile airframe

The condition for switching the control commands has been taken to be the result of comparison of the current values for the required and available acceleration.

The rule in the first case is formulated as follows: if the absolute value of the required acceleration in the control plane is greater than the absolute value of the available acceleration in the divert mode, then the switch to the opposite mode takes place. The equation with acceleration-based determination of control commands for the control plane takes the form

$$
\begin{aligned}
& \mathbf{u}_{\kappa}=\left[\begin{array}{c}
\kappa_{C} \\
\kappa_{T}
\end{array}\right]=\left[\begin{array}{c}
\eta_{1} \dot{\lambda}_{m}+\eta_{2} \ddot{\lambda}_{m} \\
\kappa_{T}
\end{array}\right] \\
& \kappa_{T}=\left\{\begin{array}{ccc}
-\eta_{1} \dot{\lambda}_{m}-\eta_{2} \ddot{\lambda}_{m} & \text { for } & \left|a_{r e q}\right|>\left|a_{a v}^{D}\right| \\
\eta_{1} \dot{\lambda}_{m}+\eta_{2} \ddot{\lambda}_{m} & \text { for } & \left|a_{r e q}\right| \leqslant\left|a_{a v}^{D}\right|
\end{array}\right.
\end{aligned}
$$

where $a_{a v}^{D}$ is the available acceleration in the divert mode.

In the second case, an extended acceleration-based conversion model is used, where the values of required acceleration are compared with the values of available acceleration in the divert and canard-control mode. If the absolute value of the required acceleration in the considered control plane is greater than the absolute value of the available acceleration in the divert mode, the switch to canard control takes place. If the absolute value of the required acceleration also exceeds the absolute value of the available acceleration in the canard-control mode, the switch to the opposite mode takes place. In the extended acceleration-based conversion model, Eq. $(4.4)_{2}$ for the control command $\kappa_{T}$ is replaced by

$$
\kappa_{T}=\left\{\begin{array}{lll}
-\eta_{1} \dot{\lambda}_{m}-\eta_{2} \ddot{\lambda}_{m} & \text { for } & \left|a_{r e q}\right|>\left|a_{a v}^{C}\right| \\
0 & \text { for } & \left|a_{a v}^{D}\right|<\left|a_{r e q}\right| \leqslant\left|a_{a v}^{C}\right| \\
\eta_{1} \dot{\lambda}_{\varepsilon}^{m}+\eta_{2} \ddot{\lambda}_{\varepsilon}^{m} & \text { for } & \left|a_{r e q}\right| \leqslant\left|a_{a v}^{D}\right|
\end{array}\right.
$$

where $a_{a v}^{C}$ is the available acceleration in the canard-control mode.

Equations (4.4) and (4.5) indicate that in the present dual-control system, the canard fins are assigned a primary role. The tail fins regulate the resulting aerodynamic control moment depending on both the relative position of the missile and the target, and on the current state of the variables in the homing loop. 
It is assumed that the control commands are overlaid with white noise with Gaussian distribution modelling the fluctuating interference caused by the noise from on-board devices and the environment. The resulting signal is limited by the saturation function

$$
\operatorname{sat}(\kappa)= \begin{cases}1 & \text { for } \quad \kappa>1 \\ \kappa & \text { for } \quad|\kappa| \leqslant 1 \\ -1 & \text { for } \quad \kappa<-1\end{cases}
$$

where $\kappa$ is the control command in the general meaning.

\section{Simulation of the guidance process}

The modelled missile guidance loop consists of systems with highly differentiated dynamics and event rates, including the rate of electromagnetic wave propagation. For these reasons, different methods of numerical integration have been used in forming approximate differential equations for elements of the guidance loop. For the elements subject to a negative feedback loop, Euler's approximations are generally sufficient, given an appropriate choice of the integration step size. Similarly, central-difference formulas computed in an open-loop system have been used to approximate the motion of the target. The proposed command formulas were examined for the terminal guidance phase. A comparison of guidance processes was made for the following missile models: a) dual-control missile No. 1 with the command formula described by equations (4.4); b) dual-control missile No. 2 with the command formula described by equations $(4.4)_{1}$ and (4.5); c) dual-control missile No. 3 with tail-fin control, with the following conversion formula

$$
\mathbf{u}_{\kappa}=\left[\begin{array}{l}
\kappa_{C} \\
\kappa_{T}
\end{array}\right]=\left[\begin{array}{c}
0 \\
-\eta_{1} \dot{\lambda}_{m}-\eta_{2} \ddot{\lambda}_{m}
\end{array}\right]
$$

The following values were used as quantity indices of the guidance process: a) the value of the expression in (4.3); and b) the miss distance $d$ defined as

$$
d=\sqrt{\left(x_{E}-x\right)^{2}+\left(y_{E}-y\right)^{2}}
$$

at the instant at which the closing velocity $V_{C}$ changed the sign.

It is assumed that the missiles have no thrust during the endgame. The following values of variables have been used in the simulation: $m=100 \mathrm{~kg}, I=35 \mathrm{kgm}^{2}, S_{B}=0.67 \mathrm{~m}^{2}, S_{C}=S_{T}=$ $0.06 \mathrm{~m}^{2}, l_{B}=0.2 \mathrm{~m}, l_{C}=l_{T}=1.36 \mathrm{~m}, \tau_{C}=\tau_{T}=0.01 \mathrm{~s}, k_{S}=1, \tau_{S}=0.01 \mathrm{~s}, \zeta=1.3 \mathrm{rad}$, $\tau_{E}=0.1 \mathrm{~s}$.

\section{Scenario 1}

The missiles are aimed at a non-maneuvering aerial target (Fig. 5). At the time $t=0$, the target is located at the pitch angle $\theta_{E}=0^{\circ}$ and is moving at $V_{E}=400 \mathrm{~m} / \mathrm{s}$ at a height of $1500 \mathrm{~m}$ and a distance of $3000 \mathrm{~m}$ from the initial position of the missiles. At $t=0$, the missiles have a height of $1000 \mathrm{~m}$, the pitch angle of the airframe is $\vartheta=5^{\circ}$, the pitch angle of the velocity vector is $\theta=5^{\circ}$, and the initial velocity is $V=1000 \mathrm{~m} / \mathrm{s}$. The time of the simulation is $t_{\text {sim }}=2.5 \mathrm{~s}$.

In the simulation, missiles 1-3 intercept the target at the time $t_{1}=t_{2}=t_{3}=2.23 \mathrm{~s}$. The mean values of the quantity indices from 100 sample runs of the scenario are given in Table 1 , where

$$
\bar{d}=\frac{1}{100} \sum_{i=1}^{100} d_{i} \quad \bar{J}=\frac{1}{100} \sum_{i=1}^{100} J_{i}
$$




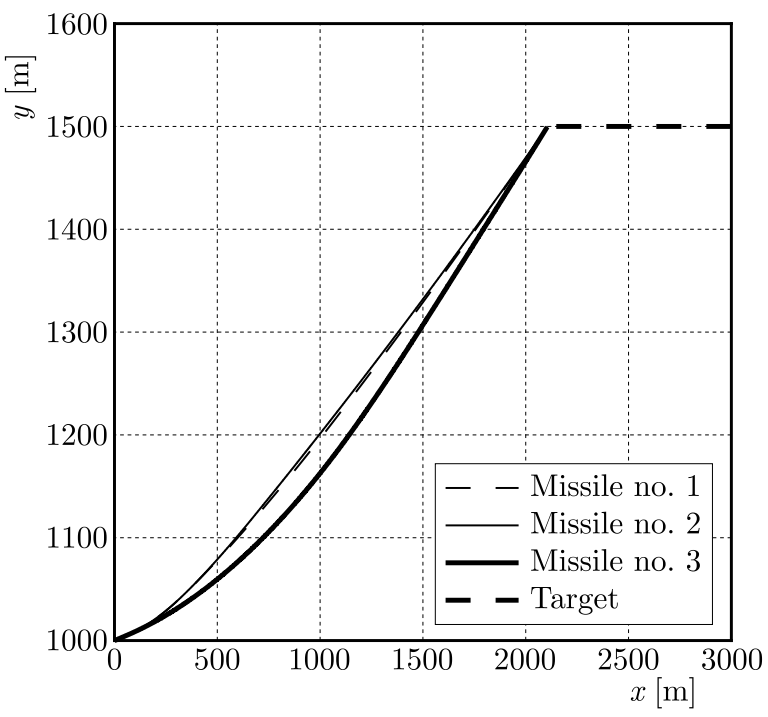

Fig. 5. Missile and target trajectories

Table 1. Guidance results

\begin{tabular}{|c|c|c|}
\cline { 2 - 3 } \multicolumn{1}{c|}{} & $\bar{d}$ & $\bar{J}$ \\
\hline \hline Missile No. 1 & 0.0054 & 5.18 \\
\hline Missile No. 2 & 0.0013 & 3.49 \\
\hline Missile No. 3 & 0.0385 & 9.44 \\
\hline
\end{tabular}

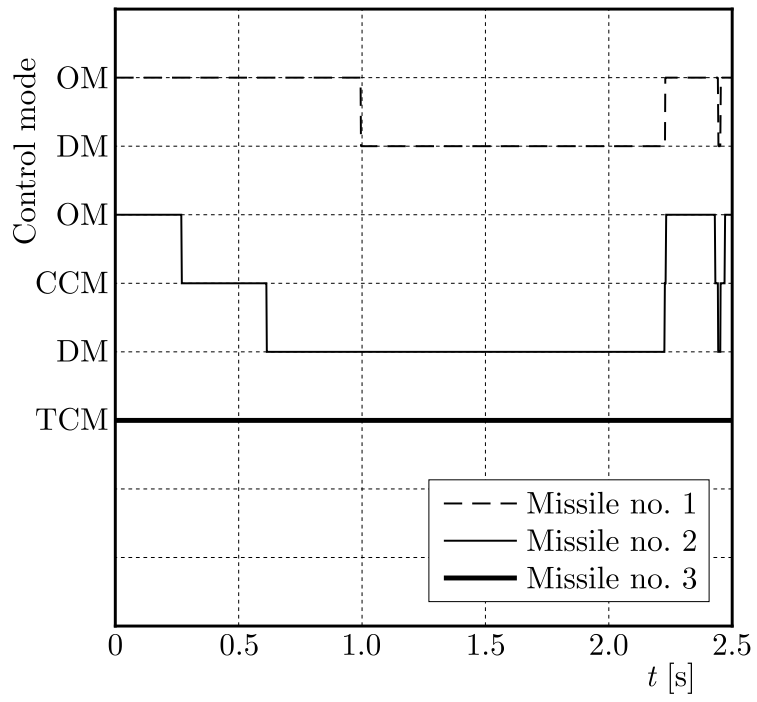

Fig. 6. Control mode comparison for a sample run (OM - opposite mode, DM - divert mode, CCM - canard-control mode, TCM - tail-fin control mode)

Figures 6 and 7 illustrate selected results from one of the simulation tests.

The analysis of the guidance process indicates that more favorable working conditions for the seeker are attained in the case of missiles No. 1 and No. 2 than in the case of missile No. 3: the angular rates of the line of sight stabilize at around $0.5 \mathrm{deg} / \mathrm{s}$ (Fig. 7a), while the missile airframes in the divert mode move at angles of attack of approximately zero (Fig. $7 \mathrm{~b}$ ). In the case of missile No. 2, the conditions for transition to the divert mode are attained $0.4 \mathrm{~s}$ earlier (Fig. 6) using an indirect control mode (canard-control mode). This leads to the most favorable stabilization index value for seeker working conditions, and the smallest average miss distance among the studied missiles (cf. Table 1). 
(a)

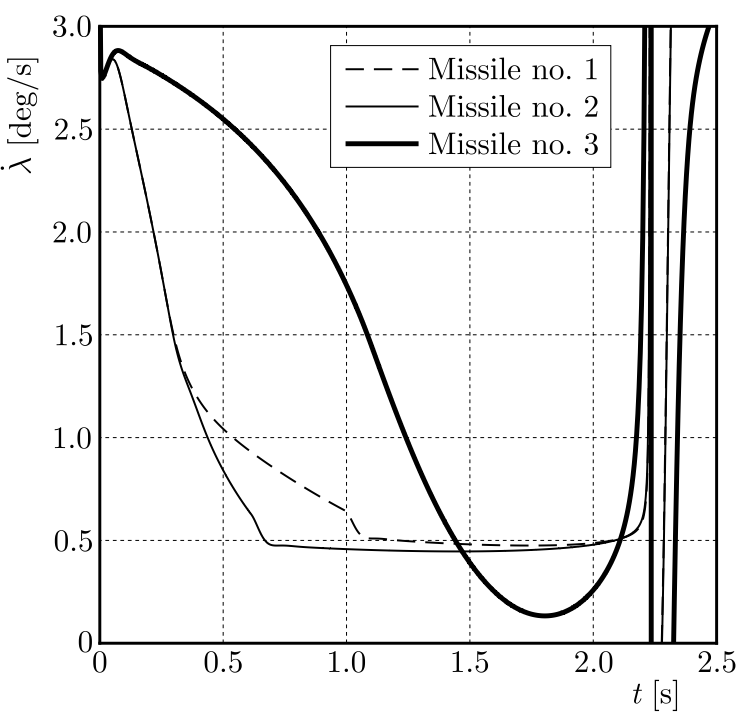

(b)

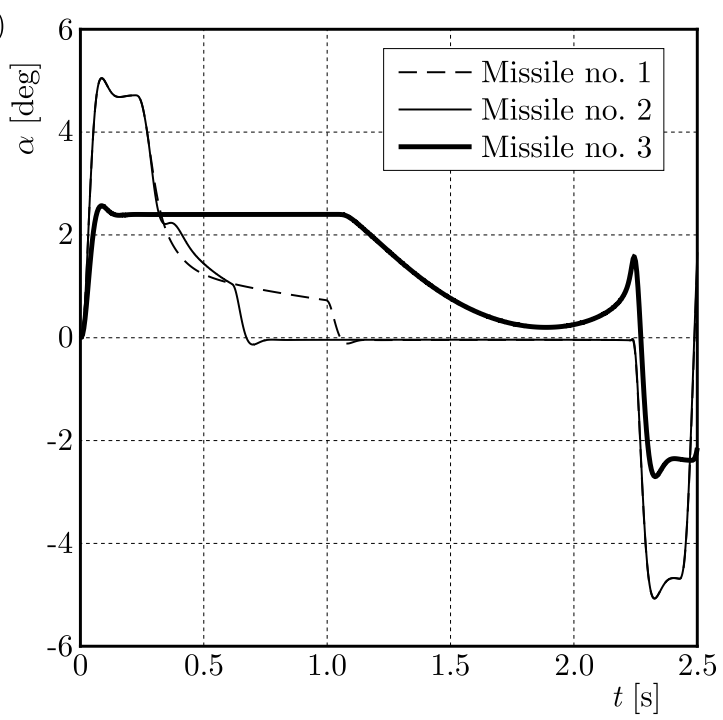

Fig. 7. (a) Angular rates of the line of sight, (b) angles of attack of the missile airframes

\section{Scenario 2}

The missiles are aimed at a fast aerial target maneuvering with longitudinal acceleration $a_{E}^{L}= \pm 1 \mathrm{~g}$ and lateral acceleration $a_{E}= \pm 10 \mathrm{~g}$ (Fig. 8). At the time $t=0$, the target is located at the pitch angle $\theta_{E}=5^{\circ}$ and is moving at $V_{E}=350 \mathrm{~m} / \mathrm{s}$ at a height of $800 \mathrm{~m}$ and a distance of $3000 \mathrm{~m}$ from the initial position of the missiles. At $t=0$, the missiles are at a height of $1000 \mathrm{~m}$, the pitch angle of the airframe is $\vartheta=0^{\circ}$, the pitch angle of the velocity vector is $\theta=0^{\circ}$, and the initial velocity is $V=1000 \mathrm{~m} / \mathrm{s}$. The time of the simulation is $t_{s i m}=2.5 \mathrm{~s}$.

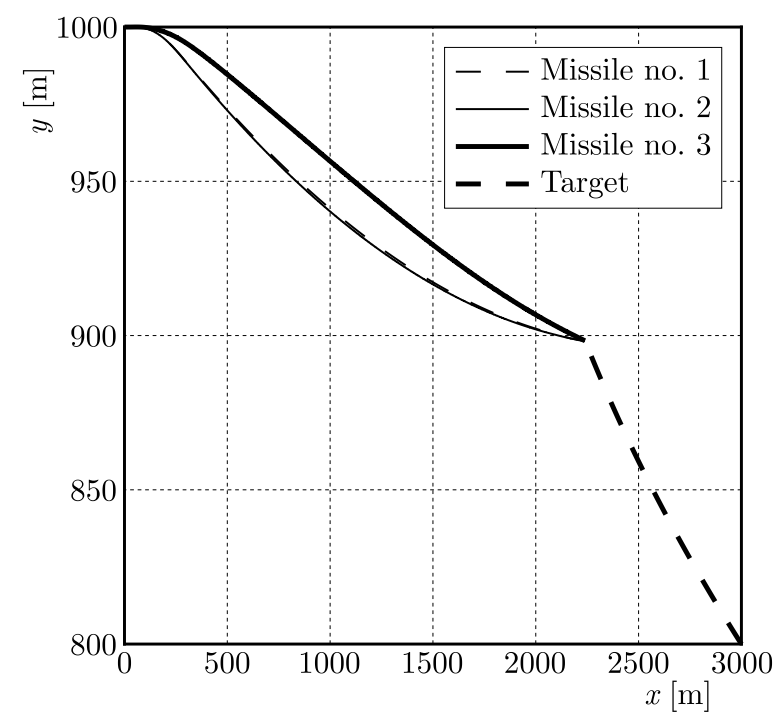

Fig. 8. Missile and target trajectories

The missiles intercept the target at the following times: $t_{1}=2.34 \mathrm{~s}$ for missile No. 1 , $t_{2}=2.33 \mathrm{~s}$ for missile No. 2 , and $t_{3}=2.32 \mathrm{~s}$ for missile No. 3 . The mean values of the quantity indices from 100 sample runs of the scenario are given in Table 2. Figures 9 and 10 illustrate selected results from one of the simulation tests.

In the case of missile No. 2, the conditions for transition to the divert mode are attained most rapidly using the indirect control mode (Fig. 9). In the case of both missile No. 1 and No. 2, it is necessary to switch to other control modes later in the guidance process: the opposite mode in 
Table 2. Guidance results

\begin{tabular}{|c|c|c|}
\cline { 2 - 3 } \multicolumn{1}{c|}{} & $\bar{d}$ & $\bar{J}$ \\
\hline \hline Missile No. 1 & 0.2663 & 67.58 \\
\hline Missile No. 2 & 0.1054 & 61.09 \\
\hline Missile No. 3 & 2.8760 & 73.73 \\
\hline
\end{tabular}

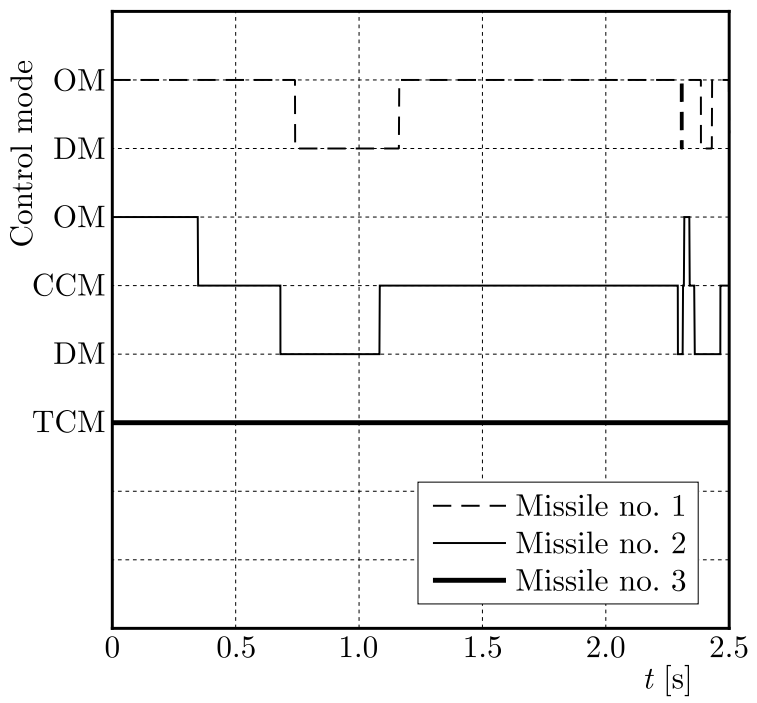

Fig. 9. Control mode comparison for a sample run (OM - opposite mode, DM - divert mode, CCM - canard-control mode, TCM - tail-fin control mode)

(a)

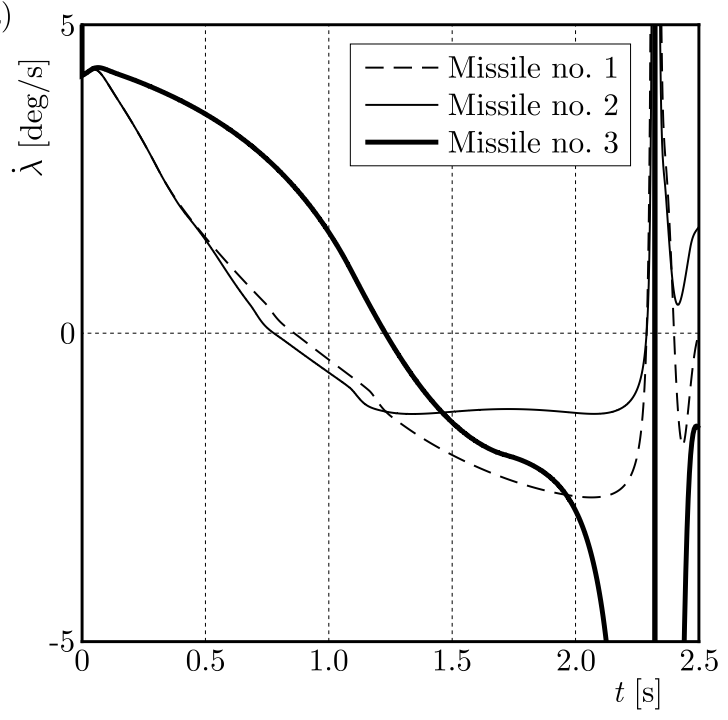

(b)

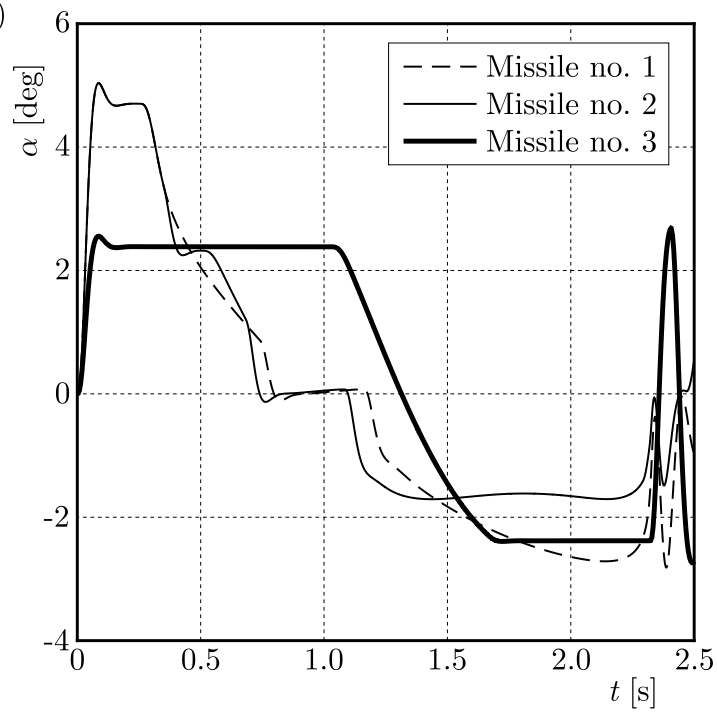

Fig. 10. (a) Angular rates of the line of sight, (b) angles of attack of the missile airframes

the case of missile No. 1, and the canard-control mode in the case of missile No. 2. However, the conditions for transitioning to the divert mode are still satisfied immediately before the strike on the target. This makes it possible to reduce the angular rate of the line of sight (Fig. 10a) leading to a reduction in the miss distance (cf. Table 2). The opposite situation occurs in the case of missile No. 3 in the tail-fin control mode: as the distance to the target decreases, the angular rate of the line of sight continues to increase, reaching a value above $300 \mathrm{deg} / \mathrm{s}$ at the moment of strike. The average miss distance of missile No. 3 is larger by an order of magnitude than that of missiles No. 1 and No. 2. 


\section{Conclusions}

The proposed control method provides a wide range of possibilities for reacting to changing conditions of an aerial combat situation.

If the target is such that the missile is not required to perform significant maneuvering, it can be guided in the divert mode with angles of attack close to zero. The use of the divert mode enables the angular rates of the missile airframe to be matched with the angular rates of the missile velocity vector, which is preceded by a short and gentle transitional process. Advantages of this mode of control include its favorable effect on the operating conditions of the sensor elements and a reduction in energy losses resulting from oscillations of the airframe. However, the limited ability to achieve normal accelerations means that guidance using the divert mode exclusively may prove insufficient to complete the task.

When the required values of normal acceleration are such that the divert mode is insufficient, the guidance can be performed using opposing deflections of the fins. This mode of control exhibits all characteristic features of control using traditional configurations (tail fins and canards) with the difference being that it enables higher aerodynamic control moments to be attained, which may be advantageous when the aerial situation requires a maneuver involving significant acceleration. The basic drawback is that the achievement of these accelerations is associated with sudden large reactions of the missile airframe, which disrupts the operating conditions of the seeker, increasing the percentage error in the control signals.

An advantage is found to result from the use of extended acceleration-based conversion with an additional control mode - canard control - as an intermediate mode between the divert and opposite modes. This enables larger accelerations to be achieved than in the case of the divert mode but, at the same time, generates lower values of the angular rates and angles of attack than the opposite mode, with resultant tactical advantages.

\section{References}

1. Gapiśski D., KrzYsZTofik I., 2014, The process of tracking an air target by the designed scanning and tracking seeker, Proceedings of 15th International Carpathian Control Conference, ICCC 2014, Velke Karlovice, Czech Republic, 129-134

2. Grycewicz H., Mosiewicz R., Pietrasieński J., 1984, Radio Control Systems (in Polish), Military University of Technology, Warsaw

3. Hua W.H., Meng Q.L., Zhang J.P., Zhang Y.J., 2016, Differential game guidance law for dual and bounded controlled missiles, Journal of Beijing University of Aeronautics and Astronautics, 42, 9, 1851-1856

4. Hull D.G., Speyer J.L., Burris D.B., 1990, Linear-quadratic guidance law for dual control of homing missiles, Journal of Guidance, Control and Dynamics, 13, 1, 137-144

5. Ibarrondo F.B., SANZ-ArÁnguez P., 2016, Integrated versus two-loop guidance-autopilot for a dual control missile with high-order aerodynamic model, Proceedings of the Institution of Mechanical Engineers, Part G: Journal of Aerospace Engineering, 230, 1, 60-76

6. Idan M., Shima T., Golan O.M., 2007, Integrated sliding mode autopilot-guidance for dual control missiles, Journal of Guidance, Control and Dynamics, 30, 4, 1081-1089

7. OCHI Y., 2003, Design of a normal acceleration and angle of attack control system for a missile having front and rear control surfaces, Transactions of the Japan Society for Aeronautical and Space Sciences, 51, 598, 621-627

8. Ochi Y., Kanai K., Saka N., Imado F., Kuroda T., 1991, A design method of normal acceleration control system for a missile with front and rear control surfaces, Proceedings of Navigation and Control Conference, New Orleans LA, USA, 196-207 
9. Schroeder W., 2001, Dual-control scheme for improved missile maneuverability, Patent No. US RE37,331 E

10. Shima T., Golan O.M., 2006, Bounded differential games guidance law for dual-controlled missiles, IEEE Transactions on Control Systems Technology, 14, 4, 719-724

11. Shima T., Golan O.M., 2007, Linear quadratic differential games guidance law for dual controlled missiles, IEEE Transactions on Aerospace and Electronic Systems, 43, 3, 834-842

12. Shtessel Y.B., Tournes C.H., 2009, Integrated higher-order sliding mode guidance and autopilot for dual-control missiles, Journal of Guidance, Control and Dynamics, 32, 1, 79-94

13. Siouris G.M., 2004, Missile Guidance and Control Systems, Springer, New York

14. YAN H., Ji H., 2012, Integrated guidance and control for dual-control missiles based on small-gain theorem, Automatica, 48, 10, 2686-2692

15. Yanushevsky R., 2007, Modern Missile Guidance, 1st Edition, CRC Press, New York

16. Zarchan P., 2012, Tactical and Strategic Missile Guidance, 6th Edition, AIAA, Reston 